\title{
A SPACE SCALAR APPROXIMATION TO THE PARTICLE-HOLE INTERACTION IN THE 2s-1d SHELL *
}

\author{
K. T. HECHT, P. J. ELLIS ** and T. ENGELAND*** \\ Physics Department, The University of Michigan, Ann Arbor, Michigan, USA
}

Received 5 July 1968

\begin{abstract}
It is shown that the diagonal matrix elements of the Gillet particle-hole interaction can be well reproduced by retaining only those terms which are space scalars.
\end{abstract}

There has been much interest in the accurate location of the energy positions of relatively complicated particle-hole states in light nuclei. Most of the recent successful calculations [1-5] have been based on the weak coupling model and have followed the approach of Bansal and French [1] whereby the major portion of the energy is taken from observed binding energies and only the particle-hole interaction energy needs to be calculated. Bansal and French point out that only the space-spin monopole or scalar part of the particle-hole interaction can make a contribution to the diagonal matrix elements if either of the particle or hole configurations are coupled to $J=0$. In this case the particle-hole interaction (except for the Coulomb contribution) can be rcpresented accurately by

$$
H_{\mathrm{ph}}=-a+b t_{\mathrm{p}} \cdot t_{\mathrm{h}} \text {. }
$$

Zamick [2] has pointed out that the interaction of eq. (1) gives the major part of the particle-hole interaction energy even in those cases where neither the particle nor the hole configurations are coupled to $J=0$. Despite its extreme simplicity, eq. (1) can be used with remarkable success to give a rough idea of the location of the particle-hole states $[2,5]$.

It is the purpose of this note to show that a significant improvement can be achieved in many cases if the particle-hole interaction is taken to include the simplest space (rather than space-spin) scalar parts of the interaction. Such an interaction can be represented by

$$
H_{\mathrm{ph}}=-a+b t_{\mathrm{p}} \cdot t_{\mathrm{h}}+c s_{\mathrm{p}} \cdot s_{\mathrm{h}}+d\left(s_{\mathrm{p}} \cdot s_{\mathrm{h}}\right)\left(t_{\mathrm{p}} \cdot t_{\mathrm{h}}\right)
$$

and may be particularly relevant for nuclei near the beginning of the $2 \mathrm{~s}-1 \mathrm{~d}$ shell where the spacial symmetry (or Wigner supermultiplet) numbers may be good quantum numbers for both the particle and hole configurations. The calculation of the matrix elements of (2) in general algebraic form has been facilitated by the recent calculation of the SU(4) (Wigner supermultiplet) Clebsch-Gordan coefficients which are needed for the calculation of space-scalar one-body and two-body operators [6]. The diagonal matrix element of eq. (2) leads to a particle-hole interaction energy of the form

$$
E_{\mathrm{ph}}^{\mathrm{int}}=a \mathrm{p}_{\mathrm{p}} \mathrm{h}_{\mathrm{h}}+b \epsilon_{2}+c \epsilon_{3}+d \epsilon_{4}
$$

where $n_{\mathrm{p}}\left(n_{\mathrm{h}}\right)=$ number of particles (holes), and where

$$
\begin{aligned}
& \epsilon_{2}=\frac{1}{2}\left[T(T+1)-T_{\mathrm{p}}\left(T_{\mathrm{p}}+1\right)-T_{\mathrm{h}}\left(T_{\mathrm{h}}+1\right)\right] \\
& \epsilon_{3}=\frac{1}{8} \frac{\left[J(J+1)-J_{\mathrm{p}}\left(J_{\mathrm{p}}+1\right)-J_{\mathrm{h}}\left(J_{\mathrm{h}}+1\right)\right]}{J_{\mathrm{p}}\left(J_{\mathrm{p}}+1\right) J_{\mathrm{h}}\left(J_{\mathrm{h}}+1\right)}\left[J_{\mathrm{p}}\left(J_{\mathrm{p}}+1\right)+S_{\mathrm{p}}\left(S_{\mathrm{p}}+1\right)-L_{\mathrm{p}}\left(L_{\mathrm{p}}+1\right)\right]\left[J_{\mathrm{h}}\left(J_{\mathrm{h}}+1\right)+S_{\mathrm{h}}\left(S_{\mathrm{h}}+1\right)-L_{\mathrm{h}}\left(L_{\mathrm{h}}+1\right)\right] \\
& \epsilon_{4}=\frac{1}{4} \epsilon_{2} \epsilon_{3} F\left(\left[\widetilde{f}_{\mathrm{p}}\right], S_{\mathrm{p}}, T_{\mathrm{p}}\right) F\left(\left[\tilde{f}_{\mathrm{h}}\right], S_{\mathrm{h}}, T_{\mathrm{h}}\right) .
\end{aligned}
$$

\footnotetext{
* Supported by U.S. Office of Naval Research, Contract Nonr. 1224(59).

** Present address: Physics Department. Rutgers University, New Brunswick. New Jersey.

*** Present address: Institute of Physics, The University of Oslo, Norway.
} 
Table 1

The Wigner supermultiplet factors

\begin{tabular}{|c|c|c|c|c|}
\hline \multirow{2}{*}{$\begin{array}{l}\mathrm{SU}(4) \\
\text { notation } \\
{[\widetilde{f}]^{(\mathrm{a})}}\end{array}$} & \multicolumn{3}{|c|}{$\begin{array}{l}\text { Symmetry labels } \\
\text { Wigner supermultiplet } \\
\text { notation. [7] }\end{array}$} & \multirow[b]{2}{*}{$F([\tilde{f}], S, T)$} \\
\hline & $P$ & $P^{\prime}$ & $P^{n}$ & \\
\hline$\left[\begin{array}{lll}y & y & 0\end{array}\right]$ (b) & $y$ & 0 & 0 & 0 \\
\hline$[y, y-1,0]$ & $\frac{1}{2} y-\frac{1}{2}$ & $\frac{1}{2}$ & $\frac{1}{2}$ & {$\left[\left(y+\frac{3}{2}\right)+2\left(S+\frac{1}{2}\right)\left(T+\frac{1}{2}\right)(-1)^{y-S-T}\right]$} \\
\hline$\left[\begin{array}{lll}y & y & 1\end{array}\right]$ & $\frac{1}{2} y-\frac{1}{2}$ & $\frac{1}{2}$ & $-\frac{1}{2}$ & $4 S(S+1) T(T+1)$ \\
\hline$\left[\begin{array}{lll}y & 0 & 0\end{array}\right]$ & $\frac{1}{2} y$ & $\frac{1}{2} y$ & $\frac{1}{2} y$ & $(y+2)$ \\
\hline$\left[\begin{array}{lll}y & y & y\end{array}\right]$ & $\frac{1}{2} y$ & $\frac{1}{2} y$ & $-\frac{1}{2} y$ & $T=S$ in this case \\
\hline$\left[\begin{array}{lll}y & 1 & 1\end{array}\right]$ & $\frac{1}{2} y$ & $\frac{1}{2} y$ & $\left(\frac{1}{2} y-1\right)$ & $\begin{array}{cc}\text { Case } 1: S=T & \text { Case } 2: S T=S(S-1) \text { or }(S-1) S \\
{[y S(S+1)-(y+2)]} & (y+2)\end{array}$ \\
\hline$[y, y-1, y-1]$ & $\frac{1}{2} y$ & $\frac{1}{2} y$ & $-\left(\frac{1}{2} y-1\right)$ & $2 S^{2}(S+1)^{2}$ \\
\hline$\left[\begin{array}{lll}y & 1 & 0\end{array}\right]$ & $\left(\frac{1}{2} y+\frac{1}{2}\right)$ & $\left(\frac{1}{2} y-\frac{1}{2}\right)$ & $\left(\frac{1}{2} y-\frac{1}{2}\right)$ & $\begin{array}{cc}\text { Case 1: } S=T & \text { Case 2: } S T=S(S-1) \text { or }(S-1) S \\
{[(y+3) S(S+1)-(y+1)]} & (y+1)\end{array}$ \\
\hline$[y y, y-1]$ & $\left(\frac{1}{2} y+\frac{1}{2}\right)$ & $\left(\frac{1}{2} y-\frac{1}{2}\right)$ & $-\left(\frac{1}{2} y-\frac{1}{2}\right)$ & $2 S^{2}(S+1)^{2} \quad 2 S^{2}$ \\
\hline
\end{tabular}

(a) $[\widetilde{f}] \equiv\left[\widetilde{f}_{1}-\widetilde{f}_{4} \cdot \widetilde{f}_{2}-\widetilde{f}_{4}, \widetilde{f}_{3}-\widetilde{f}_{4}\right]$. where $\widetilde{f}_{i}=$ number of squares in the $i$ th row of the Young tableau describing the symmetry of the spin-isospin part of the wave function.

(b) $y=$ arbitrary integer

(c) $z=\frac{P^{n}}{\left|P^{n}\right|}$.

Subscripts $\mathrm{p}(\mathrm{h})$ refer to the particle (hole) quantum numbers, and $J, T$ are the total angular momentum and isospin. The dependence on the Wigner supermultiplet quantum numbers $[\widetilde{f}]$ is given by the functions $F$, which (except for normalization factors) are Wigner coefficients for the group SU(4) $\supset$ [SU(2) $\times$ $\times \mathrm{SU}(2)]$, calculated in ref. 6 . The functions $F$ are given in table 1 for many of the simple Wigner supermultiplets of actual interest.

To investigate the approximations inherent in the use of eq. (3), the central potential of Gillet [8] has been chosen for the particle-hole interaction. The space scalar part of this potential gives $a=0.61$, $b=3.72, c=1.20, d=5.89 \mathrm{MeV}$ for the parameters of (3). Zamick [2] has used the parameters $c=d=$ $=0$. and $a=0.34, b=5.50$, or $a=0.49, b=4.91$, which are comparable. In contrast, the Kallio-Kolltveit interaction [9] which gives good results for $(\mathrm{sd})^{n}$ yields $a=1.43, b=2.71, c=1.11$, and $d=7.63$ $\mathrm{MeV}$, - clearly too strongly repulsive.

The third term of eq. (3) generally gives rather a small effect, but the fourth term may lead to important contributions to the particle-hole interaction energy in many cases. For configurations with an even number of particles or holes, however, the most common symmetries of the spin-isospin wave

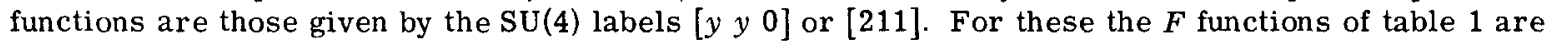
zero, and the major part of the particle-hole interaction energy is therefore given by eq. (1) as proposed by Zamick. For configurations with both $n_{\mathrm{p}}$ and $n_{\mathrm{h}}=$ odd number, (and some cases with $n_{\mathrm{p}}\left(n_{\mathrm{h}}\right)=$ $=$ even), the additional terms of eqs. (2) and (3) can lead to an improved estimate of the particle-hole interaction.

Some characteristic results are shown in table 2. We compare the matrix elements obtained using the complete Gillet potential for the particle-hole interaction to those obtained by retaining only its two-term space-spin monopole part, eq. (1), and its four-term space monopole part, eq. (3). It can be 
Table 2

Diagonal matrix elements of the particle-hole interaction. Comparison of the complete Gillet potential with its 2 term approximation (eq. (1) and its 4-term approximation (eq. (3)).

\begin{tabular}{|c|c|c|c|c|c|c|c|c|c|c|c|c|c|c|c|c|c|}
\hline \multirow[b]{2}{*}{ Nucleus } & \multirow[b]{2}{*}{$n_{\mathrm{p}}$} & \multirow[b]{2}{*}{$n_{\mathrm{h}}$} & \multirow[b]{2}{*}{$J^{\pi}$} & \multirow[b]{2}{*}{$T$} & \multicolumn{5}{|c|}{ Particle structure* } & \multicolumn{5}{|c|}{ Hole structure } & \multirow{2}{*}{$\begin{array}{l}\text { Eq. (1) } \\
\text { 2-term }\end{array}$} & \multirow{2}{*}{$\begin{array}{l}\text { Eq. (3) } \\
4 \text {-term }\end{array}$} & \multirow[b]{2}{*}{ Complete } \\
\hline & & & & & $L_{\mathrm{p}}$ & {$\left[\widetilde{f}_{\mathrm{p}}\right]$} & $s_{\mathrm{p}}$ & $T_{\mathrm{p}}$ & $J_{p}$ & $L_{\mathrm{h}}$ & {$\left[\widetilde{f}_{\mathrm{h}}\right]$} & $s_{\mathrm{h}}$ & $T_{\mathrm{h}}$ & $J_{\mathrm{h}}$ & & & \\
\hline \multirow[t]{2}{*}{$16_{O}$} & \multirow[t]{2}{*}{1} & \multirow[t]{2}{*}{1} & $0^{-}$ & 0 & \multirow[t]{2}{*}{0} & \multirow[t]{2}{*}[1]{} & \multirow[t]{2}{*}{$\frac{1}{2}$} & \multirow[t]{2}{*}{$\frac{1}{2}$} & \multirow[t]{2}{*}{$\frac{1}{2}$} & \multirow[t]{2}{*}{1} & \multirow[t]{2}{*}{ [111] } & \multirow[t]{2}{*}{$\frac{1}{2}$} & \multirow[t]{2}{*}{$\frac{1}{2}$} & \multirow[t]{2}{*}{$\frac{1}{2}$} & \multirow[t]{2}{*}{-2.18} & -0.78 & -1.21 \\
\hline & & & $1^{-}$ & 0 & & & & & & & & & & & & -2.65 & -3.09 \\
\hline \multirow[t]{2}{*}{$16_{\mathrm{O}}$} & 1 & 1 & $2^{-}$ & 0 & 2 & {$[1]$} & $\frac{1}{2}$ & $\frac{1}{2}$ & $\frac{5}{2}$ & 1 & {$[111]$} & $\frac{1}{2}$ & $\frac{1}{2}$ & $\frac{1}{2}$ & -2.18 & -1.52 & -0.61 \\
\hline & & & $3^{-}$ & 0 & & & & & & & & & & & & -2.65 & -3.00 \\
\hline${ }^{16} \mathrm{O}$ & 2 & 2 & $2^{+}$ & 0 & 3 & {$[2]$} & 1 & 1 & 4 & 1 & {$[222]$} & 1 & 1 & 2 & -5.00 & -10.17 & -9.03 \\
\hline & & & $3^{+}$ & 0 & & & & & & & & & & & & -8.62 & -7.94 \\
\hline & & & $4^{+}$ & 0 & & & & & & & & & & & & -6.55 & -6.08 \\
\hline & & & $5^{+}$ & 0 & & & & & & & & & & & & -3.96 & -3.61 \\
\hline & & & $6^{+}$ & 0 & & & & & & & & & & & & -0.86 & -1.28 \\
\hline $16_{\mathrm{O}}$ & 3 & 3 & $2^{-}$ & 0 & 2 & [111] & $\frac{1}{2}$ & $\frac{1}{2}$ & $\frac{5}{2}$ & 1 & [1] & $\frac{1}{2}$ & $\frac{1}{2}$ & $\frac{1}{2}$ & 2.71 & 3.36 & 3.10 \\
\hline & & & $3^{-}$ & & & & & & & & & & & & & 2.24 & 1.08 \\
\hline${ }^{17} \mathrm{O}$ & 3 & 2 & $\frac{1}{2}+$ & $\frac{1}{2}$ & 2 & [111] & $\frac{1}{2}$ & $\frac{1}{2}$ & $\frac{5}{2}$ & 1 & [222] & 1 & 1 & 2 & -0.05 & 1.17 & 0.32 \\
\hline & & & $\frac{3}{2}^{+}$ & $\frac{1}{2}$ & & & & & & & & & & & & 0.91 & 0.66 \\
\hline & & & $\frac{5}{2}^{+}$ & $\frac{1}{2}$ & & & & & & & & & & & & 0.47 & 0.48 \\
\hline & & & $\frac{7}{2}^{+}$ & $\frac{1}{2}$ & & & & & & & & & & & & -0.14 & -0.64 \\
\hline & & & $\frac{9}{2}+$ & $\frac{1}{2}$ & & & & & & & & & & & & -0.93 & -1.86 \\
\hline${ }^{18} \mathrm{~F}$ & 3 & 1 & $0^{-}$ & 0 & 0 & [111] & $\frac{1}{2}$ & $\frac{1}{2}$ & $\frac{1}{2}$ & 1 & [111] & $\frac{1}{2}$ & $\frac{1}{2}$ & $\frac{1}{2}$ & -0.96 & -1.76 & -2.07 \\
\hline & & & $1^{-}$ & 0 & & & & & & & & & & & & -0.69 & -1.09 \\
\hline${ }^{18} \mathrm{O}$ & 5 & 3 & $2^{-}$ & 1 & 2 & [221] & $\frac{1}{2}$ & $\frac{3}{2}$ & $\frac{5}{2}$ & 1 & {$[1]$} & $\frac{1}{2}$ & $\frac{1}{2}$ & $\frac{1}{2}$ & 4.51 & 4.94 & 5.21 \\
\hline & & & $3^{-}$ & 1 & & & & & & & & & & & & 4.21 & 3.66 \\
\hline${ }^{18} \mathrm{O}$ & 5 & 3 & $1^{-}$ & 1 & 2 & [221] & $\frac{1}{2}$ & $\frac{3}{2}$ & $\frac{5}{2}$ & 1 & [221] & $\frac{1}{2}$ & $\frac{3}{2}$ & $\frac{3}{2}$ & -1.07 & -0.85 & -0.28 \\
\hline & & & $2^{-}$ & & & & & & & & & & & & & -0.93 & -1.02 \\
\hline & & & $3^{-}$ & 1 & & & & & & & & & & & & -1.06 & -1.08 \\
\hline & & & $4^{-}$ & 1 & & & & & & & & & & & & -1.22 & -1.82 \\
\hline${ }^{20} \mathrm{Ne}$ & 5 & 1 & $2^{-}$ & 0 & 2 & {$[1]$} & $\frac{1}{2}$ & $\frac{1}{2}$ & $\frac{5}{2}$ & 1 & [111] & $\frac{1}{2}$ & $\frac{1}{2}$ & $\frac{1}{2}$ & 0.26 & 0.92 & 1.35 \\
\hline & & & $3^{-}$ & 0 & & & & & & & & & & & & -0.20 & -0.86 \\
\hline${ }^{20} \mathrm{Ne}$ & 5 & 1 & $2^{-}$ & 0 & 1 & {$[1]$} & $\frac{1}{2}$ & $\frac{1}{2}$ & $\frac{3}{2}$ & 1 & [111] & $\frac{1}{2}$ & $\frac{1}{2}$ & $\frac{1}{2}$ & 0.26 & 1.04 & 0.86 \\
\hline & & & $2^{-}$ & 0 & & & & & & & & & & & & -0.20 & -0.41 \\
\hline
\end{tabular}

* For the particles we have taken the $(\lambda \mu)$ with the highest value of the SU(3) Casimir invariant [3]. Changing $(\lambda \mu)$, with all other quantum numbers fixed, typically changes the "complete" matrix element by only about $0.3 \mathrm{MeV}$.

seen that the matrix elements of the four-term interaction differ from those of the complete interaction by only $1 \mathrm{MeV}$ in the worst cases, and often give significantly better results than the two-term case. In particular, eq. (3) reproduces quite well the $J$-dependence of the matrix elements.

Since the off-diagonal matrix elements of the particle-hole interaction may give rise to significant mixing of states [3], they must also be considered. The Gillet interaction gives the largest off-diagonal matrix elements between particle-hole states with the same space structure. These are quite well es- 
timated using the interaction (2) in those cases where a space monopole interaction can contribute. (The Wigner supermultiplet factors off-diagonal in ST are required and will be given elsewhere [6].) However, in general it is not sufficient to use the simple space monopole interaction since significant contributions to the off-diagonal matrix elements can also be obtained from the higher space-multipole com. ponents.

Despite its obvious limitations, estimates based on the interaction (2) may be used to give a rough idea of the position of the particle-hole states and may be particularly useful in surveying exotic particle-hole states to see whether they can lead to low-lying states of nuclei.

\section{References}

1. R. K. Bansal and J.B. French, Phys. Letters 11 (1964) 145.

2. L.Zamick, Phys. Letters 19 (1965) 580.

3. T. Engeland and P.J.Ellis, Phys. Letters 25B (1967) 57 ,

4. A. Arima, H.Horiuchi and T. Sebe, Phys. Letters 24B (1967) 129.

5. G.E.Brown and A.M.Green, Nucl. Phys, 85 (1966) 87.

6. S.C.Pang and K.T.Hecht, to be published.

7. M. Hamermesh, Group theory and its application to physical problems (Addison Wesley, Reading and London, 1962) p. 442.

8. V.Gillet, Nucl. Phys. 51 (1964) 410.

9. A. Kallio and K. Kolltveit, Nucl. Phys. 53 (1964) 87. 